\title{
Medición del impacto social y económico: políticas públicas de emprendímiento en Andalucía
}

\author{
José María Casado Ruiz \\ Enrique Bernal Jurado \\ Adoración Mozas Moral \\ Domingo Fernández Uclés \\ Miguel Jesús Medina Viruel
}

RESUMEN: En los últimos años hemos padecido una crisis económica que ha intensificado la preocupación de los gobiernos por corroborar la eficacia y eficiencia con la que se utilizan los fondos públicos. Esta situación se justifica (Wiesner, 2000), entre otras, por la mayor necesidad de responsabilidad y de transparencia, por el alto coste político que se sufre cuando la opinión pública estima la falta de eficiencia de los programas públicos y por la importancia de que los ajustes o correcciones fiscales sean racionales. Por otra parte, la necesidad de evaluación de los programas de desarrollo empresarial está justificada por la gran cantidad de fondos destinados a su implementación (Awasthi y Sebastián, 1996). El objetivo de este trabajo es evaluar las políticas públicas y constatar cómo, a través de la medición del impacto económico y social de los programas públicos, se puede conocer el retorno de la inversión pública a la sociedad. Tomando como caso de estudio la Junta de Andalucía, se analizará el retorno de la inversión pública en materia de emprendimiento de Andalucía Emprende, Fundación Pública Andaluza en el año 2015. Se ha utilizado la metodología coste-beneficio. Los resultados indican que el retorno a la sociedad supera la inversión realizada.

PALABRAS CLAVE: Evaluación de proyectos, coste-beneficio, política pública, valor social, monetización, Andalucía Emprende, desarrollo regional.

CLAVES ECONLIT: 022, R11, R58, M14.

Cómo citar este artículo / How to cite this article: CASADO, J.M., BERNAL, E., MOZAS, A., FERNÁNDEZ,

D. \& MEDINA, M. (2017): "Medición del impacto social y económico: políticas públicas de emprendimiento en Andalucía", CIRIEC-España, Revista de Economía Pública, Social y Cooperativa, 90, 75-102.

Correspondencia: José María Casado Ruiz, Universidad de Jaén, jmcasado@ujen.es, Doctor; Enrique Bernal Jurado, Universidad de Jaén, ebernal@ujaen.es, Doctor; Adoración Mozas Moral, Universidad de Jaén, amozas@ujaen.es, Doctora; Domingo Fernández Uclés, Universidad de Jaén, dfucles@ujaen.es, Doctor, y Miguel Medina Viruel, Universidad de Córdoba, mjmedina@ujaen.es, Doctor. 


\section{EXPANDED ABSTRACT}

\section{Measuring the social and economic impact of public policies on entrepreneurship in Andalusia}

The purpose of this study was to examine the need to assess public policies and to establish that it is possible, by measuring the economic and social impact of public programs, to establish the returns to society from public investment. Taking the example of the regional government of Andalusia, it examined the return on public investment in entrepreneurship through the Andalusian public foundation Andalucía Emprende.

In recent years, Spain has suffered an economic crisis that has intensified the concern of different levels of government to verify how effectively and efficiently public funds are being used. This situation is explained (Wiesner, 2000), among other reasons, by a greater need for accountability and transparency, owing to the high political cost when public opinion considers that public programmes are lacking in effectiveness and efficiency and to the importance of ensuring that fiscal adjustments or corrections are rational.

According to Awasthi \& Sebastián (1996), the need to assess business development programmes is justified by the large sums of money devoted to implementing them.

However, despite the existence of sophisticated methods to measure the financial situation of a company that make it possible to compare different companies and estimate their financial risks, there is no method with similar characteristics to measure social and environmental aspects (Reeder \& Colantonio, 2013; Retolaza et al., 2016). Social methods make it possible to measure the impact of business activities in society, complementing financial methods, but this impact is usually ignored by companies and is not usually valued at market prices, presenting organisations with a tremendous challenge (Lamberton, 2005; Grieco, 2015).

For this purpose, an effective way of measuring the non-financial benefits of a particular programme or organisation is to analyse its social and economic impact. In spite of the theoretical and practical importance of measuring social impact, no system has been developed to classify the measurement methods. There are various reasons for this (Maas y Liket, 2011):

- Its qualitative nature makes objective evaluation difficult

- Ascertaining the positive and negative environmental, economic and social impact of the organisations or programmes under study

- Ascertaining the short and long term effects of the organisation on society. 
In addition to the foregoing, there is a lack of consensus in the literature on the terminology to be used and on the design of evaluation methods and measurement systems (Reeder \& Colantonio, 2013). The result is that researchers are currently faced with a fuzzy set of terms, tools and techniques, designed for different purposes to those for which these impacts and long-term goals are measured.

Because of this, the literature reflects a wide variety of measurement methods, depending on the characteristics, activities and objectives of different organisations and the aspects to be assessed (Grieco, 2015). Retolaza et al. (2016) classify these methods into five main categories: impact analysis, evaluation of externalities, economic evaluation, management improvements and rating systems.

Given the stated objective of examining the Andalusian regional government's policies on entrepreneurship, implemented through Andalucia Emprende to foster entrepreneurship in a particular year, the authors believe that of the methods listed in Table 1 (see the Spanish text), those best suited to this purpose are economic (monetary) valuation ones for cost-benefit analysis. This is a traditional economics tool for performance management, adapted to include impacts on society by expressing the social costs and impacts of an investment in monetary terms.

In terms of the literature generated (Retolaza et al., 2016), 700 high-impact published works included "cost-benefit" among their keywords in 2014.

This method was applied to the Andalusian public foundation Andalucía Emprende, a non-profit public organisation, financed exclusively by public funding, through which the Andalusian regional government channels a large part of its entrepreneurship policies. It manages the Andalusian entrepreneurship centres (Centros Andaluces de Emprendimiento or CADEs), which have the mission of promoting Andalusian economic activity by developing an entrepreneurial culture, with the aim of increasing the creation of competitive companies and stable employment in the region.

Its origins go back to 1990 and the programme for a Business School for Youth Cooperatives, composed of under 25-year-olds (Mozas \& Bernal, 2007; De Pablo \& Uribe, 2009; De Pablo et al., 2010; Martí-Costa, 2010). A number of entrepreneurship-related programmes are currently underway. The foundation has an annual budget of nearly $€ 40$ million, manages 217 centres throughout Andalusia, employs a professional staff numbering 950 and has over 800 spaces in which to accommodate companies.

The cost-benefit method highlights the benefits obtained by society as a result of the free services to promote entrepreneurship provided by this public organisation to fulfil its founding objectives, and the savings and returns obtained in this way by the administration itself. The year examined was 2015 , based on the information provided by this organisation's accounts and management report for that year. 
For this purpose, Hehenberger et al. (2013) established a frame of reference, common to practically all the methods employed to measure social impact, which consists of five steps: establishing the objectives, identifying the stakeholders, measuring the results, verifying and valuing the impact and, finally, monitoring and presenting the results in the form of a report.

An analysis of the stakeholders of the foundation was therefore conducted, taking into account the benefits they receive through the activities of Andalucia Emprende and the value assigned to the outcomes. These are shown in Table 3 (see the Spanish text), with the benefits received through the activities of the organisation (the outcomes), their valuation and the weighting applied to obtain the impact.

A value was then placed on each of the outcomes generated by the stakeholders as a result of the organisation's activity. This valuation presented a considerable challenge, as there is no defined value for each of the outcomes and each stakeholder could apportion a different value to the benefits received. Therefore, reference values were taken from the statistics provided by the Andalusian statistics institute (Instituto de Estadística de Andalucía, IEA), the annual Spanish tax revenue report (Agencia Tributaria, 2016) and companies providing similar services to those offered by Andalucía Emprende.

After applying different weightings, the calculations resulted in the figures shown in Table 4 (see the Spanish text). These results indicate that the public investment of $€ 40.2$ million generated a return of €271.39 million, distributed as follows:

- Direct returns to Andalucía Emprende were $€ 0.36$ for each $€ 1$ invested.

- The return generated by companies formed in the year 2015 was $€ 1.04$ for each $€ 1$ invested.

- In terms of the higher survival rate of companies assisted by Andalucía Emprende compared to the mean rate for Andalusia, the return was $€ 4.16$ for each $€ 1$ invested.

- Indirect returns from free services and unemployment benefit savings generated $€ 1.18$ for each $€ 1$ invested.

In short, each $€ 1$ invested in Andalucia Emprende gave society a return of $€ 6.75$. It should also be mentioned that the effect of the foundation's activity in years subsequent to 2015 was not taken into account and that many services provided by this organisation could not be valued in financial terms, so the return on the investment would in fact be greater. Consequently, the value calculated in this study is less than the real value.

A priori, this indicates good management of public funds, as the figure generated is much higher than that invested, without entering into any valuation of the opportunity cost of investing these funds in a different project.

The main limitation on this study lies in the characteristics of the method employed, owing to the difficulty of placing a value on certain outcomes generated by the organisation's activities and to the existence of intangibles that could not be monetised. 
Future lines of research should focus on methodological improvements that would contribute to reaching consensus on a method that companies can use in a generic and systematic way, as in accountancy, with a series of accepted international standards that would allow all the social and environmental value that companies generate to be shown on their balance sheets.

KEYWORDS: Project evaluation, cost-benefit, public policy, social value, monetization, Andalucía Emprende, regional development.

\section{1.- Introducción}

En los últimos años hemos padecido una crisis económica que ha intensificado la preocupación de los distintos gobiernos por corroborar la eficacia y eficiencia con la que se utilizan los fondos públicos. Además, tanto los investigadores como las diferentes instituciones están planteando la posibilidad de optar por modelos de desarrollo socioeconómicos más sostenibles y responsables (Pérez y Valiente, 2017). De esta forma, actualmente, se analiza la Responsabilidad Social en las Administraciones Públicas, aunque se encuentra en una fase inicial, siendo importante para desarrollar una verdadera política de crecimiento y desarrollo territorial (Vallecillo y Gutiérrez, 2016).

Esta situación se justifica (Wiesner, 2000), entre otras, por la mayor necesidad de responsabilidad y de transparencia, por el alto coste político que se sufre cuando la opinión pública estima la falta de eficacia y eficiencia de los programas públicos y por la importancia de que los ajustes o correcciones fiscales sean racionales. En este último caso, partiendo de un necesario conocimiento de las políticas y los programas desarrollados por el sector público y de su impacto, se realizan recortes de aquellas medidas que se consideran prescindibles, según las prioridades establecidas.

Con la evaluación de un programa público se pretende demostrar el acierto del mismo y de los mecanismos puestos en marcha para su ejecución, comprobar su idoneidad y la eficacia en la consecución de los objetivos inicialmente planteados, justificar los gastos presupuestados y ejecutados, servir de apoyo para su extrapolación a otras regiones, justificar su bondad y/o retrasar la implantación de uno nuevo en espera de una justificación de eficacia o eficiencia que lo respalde (Planas, 2005).

Por otro lado, si bien existen sofisticados métodos de medición de la situación financiera de una empresa, que permiten comparar a varias empresas entre sí y valorar sus riesgos financieros, no existe, sin embargo, un método con características similares para la medición de aspectos sociales y medioambientales (Reeder y Colantonio, 2013; Retolaza et al., 2016). Los métodos sociales permiten medir 
el impacto de las actividades empresariales en la sociedad, sirviendo de complemento a los métodos financieros, aunque este impacto suele ser ignorado por las empresas y no suele ser valorado a precios de mercado, constituyendo un enorme desafío para las organizaciones (Lamberton, 2005; Grieco, 2015).

El objetivo de este trabajo es analizar la necesidad de evaluación de las políticas públicas y constatar cómo, a través de la medición del impacto económico y social de los programas públicos, se puede conocer el retorno de la inversión pública a la sociedad. Para ello, tomando como ejemplo la Junta de Andalucía, se analizará el retorno de la inversión pública en materia de emprendimiento, a través de Andalucía Emprende, Fundación Pública Andaluza1.

Para alcanzar dicho objetivo, el trabajo se ha estructurado como sigue: tras esta introducción, en el segundo apartado, se analiza la necesidad de evaluar las políticas públicas y se expone la amplia variedad de métodos para la medición del impacto económico y social, concluyendo en el método que vamos a utilizar en este artículo; en el tercero, se relatan los antecedentes de Andalucía Emprende, Fundación Pública Andaluza, para posteriormente realizar la propuesta metodológica, aplicar el método seleccionado y analizar los datos obtenidos; finalmente, en el cuarto apartado, se recogen las principales conclusiones obtenidas.

\section{2.- Necesidad de evaluación de las políticas y cálculo del impacto económico y social}

La necesidad de evaluar las políticas y los servicios públicos es, por una parte, una exigencia de la ciudadanía y, por otra, una necesidad política y de gestión. Cada vez más se reclama una mayor eficiencia y responsabilidad en la gestión y ejecución del presupuesto por los distintos actores, debido al alto grado de descentralización y limitaciones de disponibilidad de recursos existentes. Así, de acuerdo con Awasthi y Sebastián (1996), la necesidad de evaluación de los programas de desarrollo empresarial está justificada por la gran cantidad de fondos destinados a su implementación. Su mantenimiento en el tiempo debe depender de la consecución de los objetivos, a la vez que debe de justificarse en base a los costes y los ingresos generados por el programa. Estos programas deben de someterse a renovaciones y restructuraciones en función de las necesidades de los emprendedores potenciales. 
Estos procesos de evaluación del sector público se pueden enfocar desde distintas perspectivas (Ospina, 2001): en un nivel micro, como gestión de recursos humanos, realizando una evaluación del desempeño del personal que trabaja en el sector público para cumplir su cometido; en un nivel intermedio o meso, a través de la evaluación de la planificación estratégica, realizándola sobre las entidades encargadas de proporcionar servicios públicos o implementar determinadas políticas de los programas del gobierno; en un nivel macro, mediante la evaluación de los programas con distintas entidades implicadas y, en un nivel superior, la acción del gobierno como institución comprometida con una serie de políticas públicas democráticas que constituyen un pacto con los ciudadanos. En este artículo nos centraremos en un nivel meso mediante el análisis de una organización como es Andalucía Emprende, encargada del desarrollo de la ejecución de políticas de fomento del emprendimiento del gobierno de la Junta de Andalucía.

Los objetivos que se persiguen con la evaluación de las políticas y servicios públicos son variados. Siguiendo a Stufflebeam y Shinkfield (1993), se podrían resumir en los siguientes:

- Perfeccionamiento o mejora. Se busca prestar un servicio de la mayor calidad posible, siendo muy importante el resultado, debiendo adaptarse lo máximo posible a las necesidades de las personas a las que va destinado.

- Responsabilidad o rendimiento de cuentas (Vedung, 1997). Consiste en comprobar si los distintos agentes implicados han realizado su trabajo adecuadamente, permitiendo realizar juicios de valor sobre la asignación de los recursos. Se pueden describir cuatro perspectivas distintas:

- política, en la que la evaluación se rige como un instrumento en manos de la clase política para poder comprobar cómo se están ejecutando las tareas asignadas.

- técnico-organizativa, de forma que el personal directivo del organismo encargado de la ejecución de la política controla al personal subordinado en su desempeño.

- ciudadanos, centrada en el interés de este colectivo por conocer la responsabilidad de la representación política.

- clientes, que tiene su importancia en la determinación de la equidad y la satisfacción del servicio.

- Ejemplificación. Aunque las evaluaciones se diseñan para valorar un programa o una política determinada es importante que en su diseño se tenga en cuenta que también sirven para generar un conocimiento científico que puede ser aplicable a la implementación de otros programas o políticas, para iluminar investigaciones concretas u otras cuestiones teóricas.

Para ello, una forma efectiva de medir los beneficios no financieros de un determinado programa o entidades es mediante el análisis de su impacto económico y social. Este análisis facilita la toma de mejores decisiones y aumenta el beneficio económico de la inversión en objetivos sociales y/o ambientales. Pero, para ello, es necesario adoptar un enfoque cuantitativo para la evaluación de pro- 
gramas sociales en funcionamiento, predecir el retorno de las inversiones y planificar estrategias de programas a largo plazo (Brest et al., 2009). Esta información es demandada principalmente por tres actores (Arvidson et al., 2010): el sector público, financiadores de entidades sin ánimo de lucro y dichas entidades, estas últimas para demostrar su valor, a su público y a potenciales financiadores.

Pese a esta importancia teórica y práctica de medición del impacto social de los programas o de las organizaciones, no existe un sistema de categorización de métodos para su medición. Las razones son diversas (Maas y Liket, 2011): por un lado, la dificultad de realizar una valoración objetiva por su naturaleza cualitativa; por otro, hay que tener en cuenta el impacto positivo y negativo de las entidades o programas objeto de estudio a través de sus diferentes dimensiones (ambiental, económica y social); $y$, finalmente, hay que tener en cuenta tanto los efectos a corto como a largo plazo de la entidad en la sociedad. A todo lo anterior, se une la falta de consenso en la literatura sobre la terminología utilizada, el diseño de métodos de evaluación y de sistemas de métricas (Reeder y Colantonio, 2013). El resultado es la existencia, en la actualidad, de un conjunto difuso de terminología, herramientas y técnicas con diferentes fines para los que se realiza su medición y sus objetivos a largo plazo. Incluso, Grabenwarter y Liechtenstein (2011) defienden que todavía no se ha conseguido que este tipo de modelos proporcionen unos resultados satisfactorios.

Como consecuencia de lo anterior, en la literatura existe una gran variedad de métodos de medición en función de las características de las distintas entidades, sus actividades, objetivos y los aspectos a evaluar (Grieco, 2015). De este modo, Retolaza et al. (2016) realizan una clasificación de los métodos utilizados alrededor del mundo, de análisis del valor social, basada en tres publicaciones (Tuan, 2008; Olsen y Galimidi, 2008; Mulgan, 2010), clasificación a la que añaden tres métodos utilizados para empresas comerciales. Estos métodos añadidos son: GRI, Reporting Integrado y RSC. Para ello, realizan una clasificación de los mismos en función de cinco categorías principales: análisis de impacto, evaluación de externalidades, valoración monetaria, mejoras de gestión y sistemas de calificación. 
Tabla 1. Métodos de medición del impacto social2

\begin{tabular}{|c|c|c|c|c|}
\hline Objetivo & Perspectiva & Orientación & Metodología & Referencias \\
\hline \multirow[t]{12}{*}{$\begin{array}{l}\text { Análisis del } \\
\text { impacto }\end{array}$} & \multirow{9}{*}{$\begin{array}{l}\text { Cuantitativa } \\
\text { (No monetaria) }\end{array}$} & & $\begin{array}{l}\text { Movement above the US } \$ 1 \\
\text { a day threshold }\end{array}$ & Bauchet y Morduch (2013) \\
\hline & & & Progress out of Poverty Index (PPI) & Progress out of poverty (2017), Velman (2009) \\
\hline & & & Dalberg approach & Dalberg (2017) \\
\hline & & & Social value metrics & Dywan et al. (2010) \\
\hline & & & SROI calculator & Daigle et al. (2004) \\
\hline & & & Matriz del bien común & López et al. (2014) \\
\hline & & & Balance social & Gallego (2012) \\
\hline & & & B - corp & Honeyman (2014) \\
\hline & & & GRI & GRI (2016) \\
\hline & \multirow{3}{*}{ Cualitativa } & Preferencias & Opinión & Retolaza et al. (2016) \\
\hline & & & Conducta & Retolaza et al. (2016) \\
\hline & & Satisfacción & Life satisfaction assessment & Retolaza et al. (2016) \\
\hline \multirow{5}{*}{$\begin{array}{l}\text { Evaluación } \\
\text { de } \\
\text { externidades }\end{array}$} & \multirow{3}{*}{ Medioambiental } & & Huella ecológica & Moffatt (2000), López y Blanco (2008) \\
\hline & & & Trust cost (PLC) & Christoffersen et al. (2013), Trucost (2015) \\
\hline & & & $\begin{array}{l}\text { Environmental Performance } \\
\text { Reporting System (EPRS) }\end{array}$ & Florman et al. (2016) \\
\hline & \multirow[t]{2}{*}{$\begin{array}{l}\text { Creación } \\
\text { de empleo }\end{array}$} & & $\begin{array}{l}\text { Real Indicators of Success } \\
\text { in Employment (RISE) }\end{array}$ & Olsen y Galimidi (2008), Retolaza et al. (2016) \\
\hline & & & SROI analysis & Olsen y Galimidi (2008), Retolaza et al. (2016) \\
\hline \multirow{11}{*}{$\begin{array}{l}\text { Evaluación } \\
\text { económica } \\
\text { (monetaria) }\end{array}$} & \multirow[t]{2}{*}{$\begin{array}{l}\text { Reducción } \\
\text { de inputs }\end{array}$} & \multirow[t]{2}{*}{$\begin{array}{l}\text { Análisis de } \\
\text { efectividad }\end{array}$} & $\begin{array}{l}\text { Análisis de efectividad } \\
\text { de costes (CEA) }\end{array}$ & $\begin{array}{l}\text { Johannesson y Weinstein (1993), } \\
\text { Pinto et al. (2008) }\end{array}$ \\
\hline & & & Coste por impacto & Center for High Impact Philanthropy (2017) \\
\hline & $\begin{array}{l}\text { Incremento } \\
\text { de inputs }\end{array}$ & & London Benchmarking Group (LBG) & LBG (2017a y b) \\
\hline & \multirow{4}{*}{$\begin{array}{l}\text { Incremento de } \\
\text { outputs (actual) }\end{array}$} & \multirow{2}{*}{$\begin{array}{l}\text { Beneficios } \\
\text { internos }\end{array}$} & Seres (McKynsei) & Garriga (2016) \\
\hline & & & Beneficio neto & Retolaza et al. (2016) \\
\hline & & \multirow{2}{*}{$\begin{array}{l}\text { Análisis coste/ } \\
\text { beneficio }\end{array}$} & Ratio de retorno interno (IRR) & Retolaza et al. (2016) \\
\hline & & & Ratio coste/beneficio (CBA) & ABD (2013), Little y Mirrlees (1969) \\
\hline & \multirow{4}{*}{$\begin{array}{l}\text { Incremento de } \\
\text { outputs (futuro) }\end{array}$} & \multirow[t]{4}{*}{ Srol } & SROI framework & Wood y Leighton (2010), Olsen y Nicholls (2005) \\
\hline & & & SROI life & Olsen y Galimidi (2008), Retolaza et al. (2016) \\
\hline & & & SROI toolkit & Olsen y Galimidi (2008), Retolaza et al. (2016) \\
\hline & & & Evaluación del impacto social (SIA) & Esteves et al. (2012) \\
\hline
\end{tabular}

2.- Algunas metodologías mantienen su nombre en inglés para facilitar su identificación cuando no existen referencias en castellano.

CIRIEC-España, Revista de Economía Pública, Social y Cooperativa 


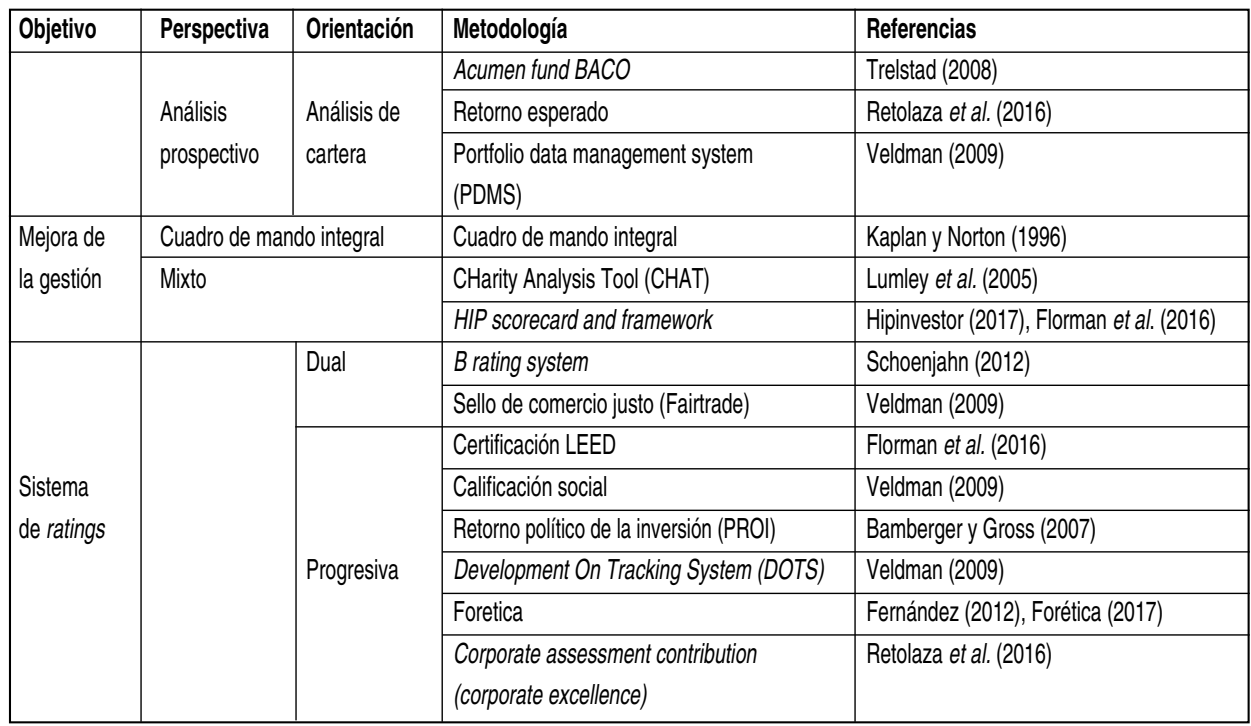

FUENTE: Elaboración propia a partir de Retolaza et al. (2016).

Si analizamos los métodos en función de su objetivo (Retolaza et al., 2016), los más desarrollados en la actualidad son los de análisis de impacto y los de sistema de ratings, pero, sin embargo, no cumplen con el objetivo planteado en esta investigación de cuantificar el impacto monetario. Por su parte, los de evaluación de externalidades están más centrados en el campo medioambiental, no adaptándose tampoco a las características del estudio y de la organización analizada. Los de mejora de la gestión están poco desarrollados metodológicamente y los de evaluación económica se encuentran más desarrollados y analizan el propósito planteado, aunque no se utilizan de forma sistemática.

Dado el objetivo de nuestro trabajo, que es el análisis de las políticas públicas de emprendimiento de la Junta de Andalucía, para el fomento del emprendimiento, ejecutadas por Andalucía Emprende, en un año concreto, entendemos que de los métodos relacionados en la tabla anterior, son los de valoración económica (monetaria) los que se adecúan mejor a dichos objetivos y, dentro de ellos, los que buscan el incremento de los outputs actuales ${ }^{3}$. Los métodos orientados a los beneficios internos, evalúan el impacto económico dentro de las organizaciones. Por ejemplo en el caso de Seres mide el impacto de RSC en la organización, en lugar de evaluar el impacto que las organizaciones tienen en la sociedad. De este modo, el análisis coste-beneficio, (Tuan, 2008; Maas y Liket, 2011; Maas, 2014)

3.- Se ha renunciado a utilizar modelos de orientación "incremento de outputs (futuros), dado que el modelo SROI, actualmente se encuentra en una etapa de evolución (Arvidson et al., 2010) y, de acuerdo con Retolaza et al. (2016), sólo es aplicable si hay inversión presente con resultados futuros, siendo difícil de aplicar si la idea es analizar el rendimiento social de las organizaciones y no de la inversión. Considerar las subvenciones como inversiones es uno de los errores más comunes de su aplicación, salvo que se traten de subvenciones de capital especificas para la inversión. 
es una herramienta económica tradicional para la gestión del rendimiento adaptado para incluir impactos en la sociedad, donde los costos y los impactos sociales de una inversión se expresan en términos monetarios y luego se evalúan de acuerdo con una o más de las siguientes tres medidas: (1) el valor actual neto, es decir, el valor agregado de todos los costes, ingresos y los impactos sociales, descontados para reflejar el mismo período contable; (2) la relación coste-beneficio, el valor descontado de los ingresos y los impactos positivos dividido por el valor descontado de los costes y los impactos negativos; y (3) la tasa interna de retorno, el valor neto de los ingresos, además de impactos expresado como un porcentaje de retorno anual sobre el importe total de la inversión.

En cuanto a la relevancia del método a nivel científico, de acuerdo con los datos aportados por Retolaza et al. (2016), en el año 2014 se realizaron en torno a 700 publicaciones de alto impacto que incluían entre las palabras clave "cost-benefit".

Por lo tanto, de los métodos analizados, nos decantamos por seguir la secuencia de trabajo del método de análisis de coste-beneficio, para analizar la empresa objeto de estudio, ya que parece ser el más adecuado para analizar el valor social mediante la diferencia entre los insumos y los productos generados durante un período de tiempo determinado, un año.

\section{3.- Andalucía Emprende, Fundación Pública Andaluza: un caso de estudio}

Andalucía Emprende, Fundación Pública Andaluza, es una organización pública, sin ánimo de lucro, que se nutre exclusivamente de fondos públicos y a través de la cual se canalizan gran parte de las políticas de emprendimiento que desarrolla el gobierno andaluz, siendo uno de los instrumentos recogidos en el anteproyecto de Ley de Fomento del Emprendimiento 4 de la Junta de Andalucía.

Esta fundación gestiona los Centros Andaluces de Emprendimiento (CADEs), cuya misión es promover la actividad económica andaluza mediante el desarrollo de la cultura emprendedora, con el objetivo de incrementar la generación de empresas competitivas y empleo estable en la región.

Sus inicios se remontan al año 1990, con el inicio del Programa de Escuela de Empresas para Cooperativas Juveniles, integradas por menores de 25 años (Mozas y Bernal, 2007; De Pablo y Uribe, 2009; De Pablo et al., 2010 y Martí-Costa, 2010). Posteriormente, en el año 1995, desaparece el término "cooperativa juvenil" del Programa y las empresas que se incuban son cooperativas o sociedades anónimas laborales conformadas mayoritariamente por menores de 30 años, posibilitando la

4.- $h$ ttp://www.juntadeandalucia.es/export/drupaljda/normativa_en_elaboracion/16/07/Texto\%20proyecto\%20ley.pdf 
excepcionalidad de alojar a autónomos. En 2004, se adscribe a la nueva Dirección General de Economía Social y Emprendedores, pasando de atender exclusivamente a cooperativas y sociedades laborales a ampliar su campo de actuación a otros tipos de formas jurídicas. En este momento se incluyen entre sus funciones "fomentar la cultura y la realidad emprendedora y empresarial, de acuerdo con la ordenación general de la economía y, en particular, con lo establecido en el ámbito de la economía social".

El éxito del programa, tanto en creación de empleo como en desarrollo económico de la región, ha llamado la atención de otras comunidades autónomas y de la Unión Europea, permitiendo su implantación en Centroamérica (De Pablo et al., 2010). Concretamente, en El Salvador se puso en marcha en mayo de 1997 en Sonsonate y Nejapa; en Panamá en junio de 2000, en Colón y San Miguelito; y en Guatemala, en Zacapa. También se llevó a Marruecos en Beni Makada, en la provincia de Tánger, y en Sidi el Mandri, en Tetuán. Estos proyectos fueron puestos en marcha mediante programas de cooperación al desarrollo de Andalucía.

Andalucía Emprende también ha asumido el cambio estratégico en las políticas de promoción empresarial, pasando de la promoción pública de "incubadoras empresariales" mediante la cesión de instalaciones a proyectos iniciales, a la disposición de espacios de trabajo compartido (coworking), fomentándose las dinámicas entre distintas iniciativas empresariales (Eizaguirre, 2016).

Actualmente se desarrollan numerosos programas relacionados con el emprendimiento, pudiendo destacarse las cifras recogidas en la tabla 2 para conocer la magnitud del trabajo realizado por la misma. 


\section{Tabla 2. Principales cifras de Andalucía Emprende, fundación pública andaluza 5}

\begin{tabular}{|l|l|rrrrrrr|}
\hline & & 2010 & 2011 & 2012 & 2013 & 2014 & 2015 & 2016 \\
\hline Presupuesto & & & $43.397 .692,84$ & $38.013 .828,90$ & $39.098 .470,73$ & 40.735 .983 & 41.063 .012 & 39.933 .419 \\
Centros & & 215 & 215 & 214 & 215 & 215 & 215 & 267 \\
\hline Alojamientos & Total & 634 & 734 & 800 & & 880 & 888 & 858 \\
empresariales & Naves & 267 & & & & 306 & 307 & 310 \\
& Oficinas & 367 & & & & 574 & 581 & 548 \\
\hline Personal & & 1.039 & 1.033 & 996 & 983 & & 947 & 946 \\
\hline Generación & Total empresas & 10.027 & 10.340 & 10.655 & 14.478 & 14.620 & 15.408 & 12.737 \\
& Econ. Social & 276 & 271 & 285 & 242 & 233 & 199 & 169 \\
& Empresario individ. & 9.041 & 9.252 & 9.378 & 12.773 & 12.666 & 13.247 & 10.613 \\
& Soc. mercantiles & 552 & 630 & 805 & 1.463 & 1.108 & 1.561 & 1.776 \\
& Otras empresas & 158 & 187 & 187 & & 613 & 401 & 179 \\
& Empleos & 13.108 & 12.652 & 14.305 & 19.633 & 19.797 & 21.182 & 17.010 \\
& Inversión (estimada) & & 121.700 .000 & 164.100 .000 & 186.900 .000 & 155.700 .000 & 168.900 .000 & 150.700 .000 \\
\hline Formación & Beneficiarios & 12.516 & 18.148 & 18.969 & 15.220 & 26.027 & 23.377 & 22.439 \\
& Horas impartidas & & 12.891 & 13.732 & 14.178 & 15.299 & 15.588 & 13.624 \\
\hline Empresas tutorizadas & 4.404 & 2.917 & 2.740 & 2.925 & 2.803 & 2.312 & 1.782 \\
Planes de desarrollo & 2.615 & 1.434 & 999 & 828 & 723 & 1.171 & 587 \\
\hline Fomento & Acciones & 1.501 & 4.531 & 6.374 & 6.568 & 6.597 & 5.921 & 5.453 \\
cultura & Beneficiarios & 104.559 & 95.023 & 130.638 & 135.371 & 269.946 & 127.235 & 121.645 \\
emprendedora & & & & & & & \\
\hline
\end{tabular}

FUENTE: Elaboración propia a partir de las memorias de actividades de Andalucía Emprende.

Como se puede observar en la tabla 2, la Fundación cuenta con un presupuesto anual que se sitúa cerca de los 40 millones de euros, gestionando 217 centros repartidos por toda Andalucía, cuenta con 950 profesionales y más de 800 alojamientos para su cesión a empresas. Aunque las cifras varían dependiendo del año analizado, las principales cifras de resultados obtenidos son la de contribuir a la creación de más de 10.000 empresas anuales y más de 14.000 empleos. También desarrolla más de 5.000 acciones de fomento de la cultura emprendedora beneficiando a más de 120.000 personas. 


\subsection{Propuesta metodológica}

Al ser una entidad sin ánimo de lucro y que recibe financiación exclusivamente pública, tanto del presupuesto de la Junta de Andalucía como de fondos comunitarios, es preciso medir el valor social que aporta a la sociedad para conocer y evaluar la eficiencia en la utilización de los fondos públicos asignados a Andalucía Emprende.

Como ya se ha mencionado, para este análisis de valor no se pueden utilizar técnicas basadas exclusivamente en parámetros financieros, ya que no reflejan el valor añadido que aporta a la sociedad, ni el ahorro de costes y el retorno de ingresos que supone para la Administración. Por lo tanto, es necesario utilizar algún método que haga visible todo ese valor añadido a los distintos stakeholders (grupos de interés de la entidad o programa objeto de análisis) y que permita evaluar la eficiencia con la que se gestionan esos fondos.

Tal y como ya se ha comentado, consideramos que la metodología basada en el análisis costebeneficio cumple con los propósitos planteados para analizar la eficacia con la que se utilizan esos fondos públicos. Esta metodología pone en valor los beneficios obtenidos por la sociedad, como consecuencia de los servicios gratuitos de fomento del emprendimiento prestados por esta entidad pública en el cumplimiento de sus fines fundacionales, y el ahorro y el retorno de ingresos que obtiene la propia Administración en el desarrollo de los mismos.

Para ello, Hehenberger et al. (2013) establecen un marco de referencia, común en prácticamente la totalidad de métodos utilizados para medir el impacto social, con cinco pasos: establecimiento de objetivos, análisis de stakeholders, medición de resultados, verificación y valoración del impacto y, finalmente, seguimiento y presentación de los resultados mediante un informe.

La cadena de creación de impacto que sigue el método que vamos a desarrollar responde al siguiente esquema:

\section{Figura 1. Cadena de creación de impacto}
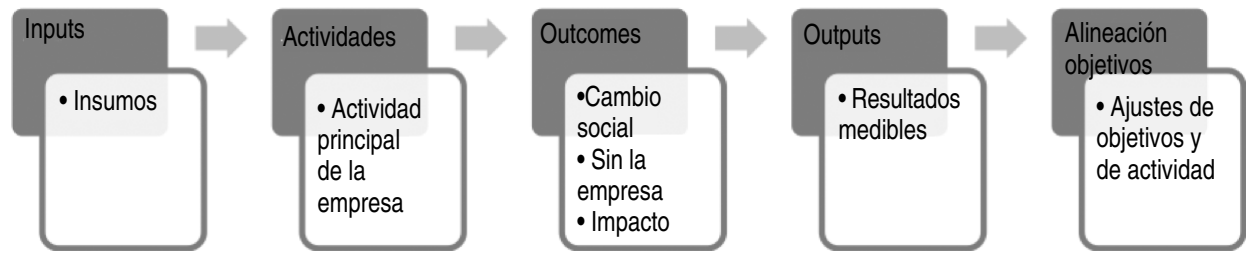

FUENTE: Clark et al. (2004). 
En el desarrollo de esta metodología nos encontramos con la dificultad de monetizar los resultados obtenidos del desarrollo de la actividad de Andalucía Emprende y su impacto, asignándoles un valor de referencia aproximado en función de las estadísticas existentes y los valores utilizados por entidades similares. Además, es importante separar los impactos que no sean fruto de la actividad de la organización de los impactos negativos que haya podido generar, teniendo en cuenta ciertos aspectos complementarios (Retolaza et al., 2016), como por ejemplo:

- El peso muerto (deadweight), es decir, el resultado obtenido sin el desarrollo del programa de emprendimiento desarrollado por esta entidad.

- Los posibles desplazamientos (displacement) existentes, es decir, beneficios que se producen a ciertos stakeholders por la actividad analizada y que afectan negativamente a otro.

- La atribución (attribution), inputs aportados por terceros que deben de ser descontados de los outputs.

- $\quad$ El decremento (drop off), deterioro en el tiempo de los outputs valorados.

\subsection{Aplicación de la metodología}

La consecución del objetivo propuesto exige la realización de un análisis evaluativo de los resultados sociales, medioambientales y económicos de la fundación Andalucía Emprende en el año 2015, partiendo de la información recogida por esta organización en las cuentas económicas de la fundación (Consejería de Hacienda y Administración Pública, 2016) y en el informe de gestión de dicho año (Andalucía Emprende, Fundación Pública Andaluza, 2016).

Para ello, una vez establecido el alcance del trabajo objeto de estudio, realizamos un análisis de los stakeholders de la fundación 6 bajo el enfoque de la Teoría de Stakeholders. A tal fin, se realiza un análisis de los mismos, desde una perspectiva ontológica, como proponen Retolaza et al. (2014), agrupándolos en función de sus intereses concretos, teniendo en cuenta las aportaciones que reciben con la actividad de Andalucía Emprende y el valor asignado a los outcomes.

Los stakeholders de Andalucía Emprende se recopilan en la tabla 3, junto con las aportaciones recibidas por la actividad de la organización (outcomes), su valoración y la ponderación aplicada para obtener el impacto.

6.- Para la realización de este análisis se ha contado con la participación de un grupo de ocho expertos, trabajadores de Andalucía Emprende, uno por cada provincia, técnicos con experiencia en el trabajo con emprendedores superior a catorce años elegidos por su disponibilidad a cooperar en el estudio. 


\section{Tabla 3. Identificación de stakeholders, outcomes, valoración, fuente y ponderación?}

\begin{tabular}{|c|c|c|c|c|c|}
\hline Stakeholders & Aportaciones recibidas & Output & Valoración & Fuente & Ponderación \\
\hline \multirow[t]{19}{*}{ Empresas } & Asesoramiento creación & Constituciones autónomos & 120,00 & \multirow{8}{*}{$\begin{array}{c}\text { Colegio } \\
\text { Economistas } \\
\text { Valladolid }(2005)^{8}\end{array}$} & \\
\hline & y desarrollo proyectos & Constituciones empresas & 600,00 & & \\
\hline & $\begin{array}{l}\text { Búsqueda de fuentes } \\
\text { de financiación }\end{array}$ & Solicitudes financiación & 120,00 & & \\
\hline & & Estudios viabilidad & & & \\
\hline & $\begin{array}{l}\text { Analilis de la viabilidad } \\
\text { de los proyectos }\end{array}$ & Estudios viabilidad & 240,00 & & \\
\hline & \multirow{7}{*}{$\begin{array}{l}\text { Mejora de la supervivencia } \\
\text { de los proyectos }\end{array}$} & Tutorizaciones & $3.400,00$ & & \\
\hline & & Plan desarrollo & 240,00 & & \\
\hline & & Plan de viabilidad & 240,00 & & \\
\hline & & Formación emp. Básica & 62,00 & \multirow{4}{*}{$\operatorname{IAAP}(2013)$} & \\
\hline & & Formación especializada & 72,00 & & \\
\hline & & Formación CRECE presencial & 62,00 & & \\
\hline & & Formación CRECE online & 27,00 & & \\
\hline & $\begin{array}{l}\text { Incremento nivel } \\
\text { emprendimiento }\end{array}$ & $\begin{array}{l}\text { Supervivencia } \\
\text { empresarial }\end{array}$ & $\begin{array}{r}\text { IRPF, IVA } \\
\text { eIS }\end{array}$ & $\begin{array}{l}\text { And. Emprende, } \\
\text { Fund. Púb. And. (2015), } \\
\text { IEA (2016 a, c y d), } \\
\text { y Agenc. Trib. (2016) }\end{array}$ & \\
\hline & Incremento inversiones & IVA inversiones & IVA & IEA (2016a) & \\
\hline & $\begin{array}{l}\text { Incremento de niveles } \\
\text { de empleo }\end{array}$ & Altas empleo & 90,00 & $\begin{array}{l}\text { Colegio Economistas } \\
\text { Valladolid (2005) }\end{array}$ & \\
\hline & Red de contactos profesionales & Encuentros empresariales & & & \\
\hline & Reconocimiento empresarial & Premios empresariales & & & \\
\hline & \multirow{2}{*}{$\begin{array}{l}\text { Alojamiento empresarial } \\
\text { gratuito }\end{array}$} & Alojamientos despachos & $2.145,00$ & \multirow{2}{*}{$\begin{array}{c}\text { Cámara Comercio } \\
\text { Toledo (2017) }\end{array}$} & \\
\hline & & Alojamiento naves & $5.352,00$ & & \\
\hline
\end{tabular}

7.- La mayoría de los output no tienen ninguna ponderación ya que si Andalucía Emprende no existe serían cero, concretamente son servicios que presta Andalucía Emprende o que no se han valorado. Las ponderaciones se aplican en los ingresos que obtiene o se ahorra la administración de las empresas que se crean.

La ponderación del $10 \%$ responde al peso muerto, es decir, se tienen en cuenta el porcentaje de empresas que no se constituirían en caso de no existir Andalucía Emprende. Este porcentaje, ante la ausencia de una cifra de referencia y la imposibilidad de conocer la realidad en ausencia de la organización, se obtiene de la consulta del grupo de ocho expertos reunidos para la determinación de los stakeholders y los outcomes.

La ponderación del $30 \%$ responde a la adaptación de los resultados obtenidos al tamaño medio de las empresas atendidas por Andalucía Emprende. Los datos estadísticos del IEA contemplan empresas de 3,9 trabajadores de media y las atendidas por Andalucía Emprende tienen una media de 1,2 .

El 25\% es el porcentaje de beneficios que obtienen las empresas de nueva creación respecto a las que están más implantadas en el mercado, de acuerdo con datos de Andalucía Emprende.

El 31,54\% es la tasa de paro en Andalucía durante el año 2015 (IEA, 2016e).

8. - Se utiliza como referencia el Colegio de Economistas de Valladolid por ser la más actualizada encontrada, ya que desde la publicación de la Ley 25/2009, de 22 de diciembre, de modificación de diversas leyes para su adaptación a la Ley sobre el libre acceso a las actividades de servicios y su ejercicio, se prohibió a los Colegios Profesionales realizar recomendaciones sobre honorarios profesionales con la excepción de los Honorarios de Tasación de Costas. 


\begin{tabular}{|c|c|c|c|c|c|}
\hline Stakeholders & Aportaciones recibidas & Output & Valoración & Fuente & Ponderación \\
\hline \multirow{6}{*}{ Ciudadanía } & $\begin{array}{l}\text { Cambio cultural hacia el } \\
\text { emprendimiento }\end{array}$ & $\begin{array}{l}\text { Acciones cultura } \\
\text { emprendedora }\end{array}$ & 120,00 & \multirow{2}{*}{$\begin{array}{c}\text { Colegio } \\
\text { Economistas } \\
\text { Valladolid (2005) }\end{array}$} & \\
\hline & Asesoramiento empresarial & Atención a usuarios & 120,00 & & \\
\hline & $\begin{array}{l}\text { Conocimiento de Estudios de } \\
\text { investigación empresarial }\end{array}$ & Estudios emprendimiento & & & \\
\hline & $\begin{array}{l}\text { Asentamiento de la población } \\
\text { en el territorio }\end{array}$ & Empleo generado & & & \\
\hline & Desarrollo rural & Empresas creadas & & & \\
\hline & Mejora posibilidades empleo & Empleo generado & & & \\
\hline \multirow{4}{*}{$\begin{array}{l}\text { Profesionales } \\
\text { Andalucía } \\
\text { Emprende }\end{array}$} & Obtención de rentas & IRPF y Seguridad Social & & & \\
\hline & Empleo público & Empleo estable & & & \\
\hline & Investigación social & Investigaciones sociales & & & \\
\hline & $\begin{array}{l}\text { Formación especializada } \\
\text { asesoramiento empresarial }\end{array}$ & Formación recibida & 72,00 & $\operatorname{IAAP}(2013)$ & \\
\hline \multirow{9}{*}{$\begin{array}{l}\text { Admon. } \\
\text { Públicas }\end{array}$} & \multirow{3}{*}{$\begin{array}{l}\text { Ingresos } \\
\text { tributarios }\end{array}$} & $A E$ & $\begin{array}{r}\text { IRPF, IVA, IS, } \\
\text { Tasas y Licencias }\end{array}$ & $\begin{array}{c}\text { Consej. Hacienda } \\
\text { y Admon. Pública (2016) }\end{array}$ & \\
\hline & & Empresas creadas & IRPF, IVA e IS & $\begin{array}{c}\text { IEA (2016 a, c y d) } \\
\text { y Agenc. Trib. (2016) }\end{array}$ & $\begin{array}{c}25 \%+10 \%+ \\
30 \%\end{array}$ \\
\hline & & Empresas supervivientes & IRPF, IVA e IS & $\begin{array}{c}\text { And. Emprende (2015) } \\
\text { IEA (2016 a, c y d), } \\
\text { y Agenc. Trib. (2016) }\end{array}$ & $30 \%$ \\
\hline & \multirow{2}{*}{$\begin{array}{l}\text { Recaudación de cotizaciones } \\
\text { sociales }\end{array}$} & Seguridad Social AE & $5.303,01$ & \multirow{2}{*}{$\begin{array}{c}\text { IEA (2016c) y MํEmpleo } \\
\text { y Seg Social (2015) }\end{array}$} & \\
\hline & & Empleos generados & $5.303,01$ & & $10 \%$ \\
\hline & \multirow[t]{2}{*}{ Ahorro prestaciones sociales } & Personal AE & $12.074,00$ & \multirow[t]{2}{*}{ IEA (2016b) } & $31,54 \%$ \\
\hline & & Empleos generados & $12.074,00$ & & $31,54 \%$ \\
\hline & Mayor cercanía a la ciudadanía & Servicios prestados AE & $27.968 .151,00$ & * & \\
\hline & Mejora de servicios públicos & Servicios prestados AE & $27.968 .151,00$ & * & \\
\hline \multirow{5}{*}{$\begin{array}{l}\text { Otras Instit. } \\
\text { (ent. financieras, } \\
\text { Universidades, } \\
\text { empresas } \\
\text { privadas y } \\
\text { Programas de } \\
\text { la AAPP) }\end{array}$} & Aparición de sinergias colaborativas & Convenios de colaboración & & & \\
\hline & Investigaciones sociales de impacto & Investigaciones sociales & & & \\
\hline & Desarrollo de programas conjuntos & Programas específicos & & & \\
\hline & $\begin{array}{l}\text { Fomento emprendimiento en } \\
\text { sectores específicos }\end{array}$ & Programas específicos & & & \\
\hline & $\begin{array}{l}\text { Difusión y tramitación de } \\
\text { convenios financieros }\end{array}$ & Solicitudes financiación & 120,00 & $\begin{array}{c}\text { Colegio } \\
\text { Economistas } \\
\text { Valladolid (2005) }\end{array}$ & \\
\hline
\end{tabular}

* Esta cifra se obtiene mediante la valoración económica de todos los servicios gratuitos prestados por Andalucía Emprende y recogidos en otras aportaciones recibidas por los distintos stakeholders.

FUENTE: Elaboración propia. 
Dado que en este caso los inputs son monetarios (fondos públicos con los que se financia la fundación), a continuación se procede a valorar cada uno de los outcomes que generan los stakeholders como consecuencia de la actividad de la organización. Esta valoración supone un gran reto, ya que no existe un valor definido para cada uno de ellos y cada stakeholder le podría otorgar un valor distinto a las aportaciones recibidas. Para realizar la valoración se han utilizado valores de referencia de las estadísticas proporcionadas por el Instituto de Estadística de Andalucía (IEA), el Informe Anual de Recaudación Tributaria (Agencia Tributaria, 2016) y empresas que prestan servicios similares a los ofertados por Andalucía Emprende.

Respecto a las cifras generadas por las empresas de nueva constitución apoyadas por Andalucía Emprende durante el año de estudio, se han adaptado los cálculos en función del tamaño medio de las empresas ${ }^{9}$ en cuanto a empleo y se ha realizado una reducción del $75 \%$ de los resultados obtenidos por Impuesto de Sociedades, IRPF de los beneficios e IVA, por ser de nueva creación y estar iniciando la actividad. Además, los resultados obtenidos se reducen en un $90 \%$ como efecto del peso muerto, ya que se entiende que, en caso de no existir esta organización, el $90 \%$ de las empresas atendidas se seguirían creando (véase nota a pie de página 6 ).

Los retornos indirectos, generados por el ahorro de prestaciones por desempleo, se calculan multiplicando la prestación por desempleo medio en Andalucía, recogida en el IEA, por el personal empleado en Andalucía Emprende más el 10\% del empleo de las empresas creadas en el año 2005 y el empleo generado por las empresas con una supervivencia mayor a la media andaluza. A la cantidad resultante, se le aplica una reducción porque, en el caso de no existir Andalucía Emprende, sólo parte del personal anteriormente mencionado se encontraría desempleado. Se estima que el porcentaje desempleado sería el mismo que la media andaluza durante el año 2015. Por lo tanto, los retornos indirectos por este concepto son el $31,54 \%$ de las cantidades que podrían recibir todo el empleo.

En el apartado de los retornos generados por el aumento de la supervivencia de las empresas creadas, se debe aclarar que actualmente no se ha concluido el estudio sobre la supervivencia de empresas en 2015 y se han utilizado los datos recogidos del año anterior, calculando los datos generados por las empresas creadas en los cinco años anteriores que sobreviven y superan a la media de Andalucía. Para ello presuponemos que el tamaño medio de las empresas, en cuanto a empleo, es el mismo que la de las empresas de nueva creación, es decir, 1,2 empleos por empresa.

\subsection{Análisis de resultados}

Con los criterios expuestos con anterioridad, se han realizado los cálculos oportunos obteniendo las cifras recogidas en la tabla 4. 


\section{Tabla 4. Resultados obtenidos del análisis coste- beneficio de andalucía emprende (AE)}

\begin{tabular}{|c|c|c|c|}
\hline & $€$ & $\%$ del total & $\begin{array}{r}\text { Retorno } \\
\text { inversión }\end{array}$ \\
\hline TOTAL INVERSIÓN AAPP EN AE & $40.200 .992,56$ & $100 \%$ & \\
\hline Gasto ejecutado AE & $40.200 .992,56$ & $100 \%$ & \\
\hline - Aportación Junta Andalucía & $35.391 .938,55$ & $88,04 \%$ & \\
\hline - Aportación Fondo Social Europeo & $4.796 .696,56$ & $11,93 \%$ & \\
\hline - Otras aportaciones públicas & $12.357,45$ & $0,03 \%$ & \\
\hline TOTAL RETORNOS & $271.388 .729,64$ & $100 \%$ & $6,75 €$ \\
\hline Retorno directo a AAPP por actividad de AE & $14.651 .570,20$ & $5,40 \%$ & $0,36 €$ \\
\hline - Seguridad social a cargo de la empresa & $8.144 .050,61$ & $3,00 \%$ & \\
\hline - Seguridad social a cuenta de trabajadores/as & $1.646 .350,98$ & $0,61 \%$ & \\
\hline - Retenciones de IRPF del empleo & $3.797 .349,76$ & $1,40 \%$ & \\
\hline - Tasas & $19.592,41$ & $0,01 \%$ & \\
\hline - IVA de compras de Andalucía Emprende & $1.044 .226,44$ & $0,38 \%$ & \\
\hline Retorno directo a AAPP de empresas creadas & $41.932 .756,64$ & $15,45 \%$ & $1,04 €$ \\
\hline - Seguridad Social & $6.231 .628,89$ & $2,30 \%$ & \\
\hline - IRPF & $1.255 .700,10$ & $0,46 \%$ & \\
\hline - Impuesto Sociedades & $73.822,13$ & $0,03 \%$ & \\
\hline - IVA de compras e inversión realizada & $34.371 .605,52$ & $12,67 \%$ & \\
\hline $\begin{array}{l}\text { Retorno directo a AAPP de empresas supervivientes } \\
\text { sobre la media andaluza (de los } 5 \text { años anteriores) }\end{array}$ & $167.369 .885,41$ & $61,67 \%$ & $4,16 €$ \\
\hline - Seguridad Social & $8.231 .049,74$ & $3,03 \%$ & \\
\hline- IRPF & $2.341 .590,77$ & $0,86 \%$ & \\
\hline - Impuesto Sociedades & $833.197,50$ & $0,31 \%$ & \\
\hline - IVA de compras & $155.964 .047,40$ & $57,47 \%$ & \\
\hline Retornos indirectos a AAPP por actividad de AE & $47.434 .517,39$ & $17,48 \%$ & $1,18 €$ \\
\hline $\begin{array}{l}\text { - Valoración económica de servicios de AE } \\
\text { - Ahorro en prestaciones por desempleo (empleo AE, empleo } \\
\text { creado por empresas y empresas supervivientes sobre media andaluza) }\end{array}$ & $\begin{array}{l}27.968 .151,00 \\
19.466 .366,39\end{array}$ & $10,31 \%$ & \\
\hline
\end{tabular}

FUENTE: Elaboración propia.

Como se puede observar, los resultados obtenidos del análisis de 2015, indican que la inversión pública realizada por importe de 40,2 millones de euros ha generado un retorno de 271,39 millones. Dichos retornos se distribuyen de la siguiente forma:

- Un 5,4\% de ese retorno obtenido (14,65 millones), corresponde a retornos directos de la propia Andalucía Emprende en concepto de Seguridad Social del personal empleado, retenciones de IRPF, tasas e IVA pagado por la propia actividad. Ello supone que de cada euro invertido en la organización, retornan 0,36 euros por estos conceptos. 
- Con relación a los retornos indirectos:

- el retorno generado por las empresas de nueva constitución del año 2015, con los ajustes mencionados anteriormente, reportan un poco más de la cantidad invertida, suponiendo un $15,45 \%$ del total. De cada euro invertido vuelve a las arcas de la Administración 1,04 euros.

- el mayor retorno recibido tiene su origen en la mayor supervivencia de las empresas apoyadas desde Andalucía Emprende en relación con la media de Andalucía. En concreto, supone 4,16 euros por cada euro invertido por la Administración, es decir, el $61,67 \%$ del retorno total.

- finalmente, los retornos indirectos, procedentes de los servicios gratuitos que presta la entidad y del ahorro de prestaciones por desempleo por parte de la Administración, suponen un $17,48 \%$ y generan 1,18 euros por cada euro invertido.

Como resumen de los datos obtenidos podemos decir que cada euro invertido en Andalucía Emprende reporta a la sociedad 6,75 euros.

Además, hay que añadir que no se han tenido en cuenta la incidencia de la actividad de la fundación en años posteriores al 2015 y también hay muchos servicios que son prestados por esta organización que no han podido ser valorados económicamente, lo que supondría un mayor retorno de la inversión. Estos servicios realizados en 2015 son los siguientes:

- Se han puesto en funcionamiento en 23 herramientas telemáticas tanto para la gestión diaria de las empresas como para la planificación de la actividad a largo plazo.

- Se ha facilitado la inserción en el mercado de trabajo de jóvenes menores de 30 años realizando prácticas en empresas alojadas en Andalucía Emprende (Programa EiLAB). Han participado 26 empresas en este programa, facilitando la inserción laboral de 43 jóvenes, de los que 18 han sido contratados y 25 han realizado prácticas formativas.

- Se han desarrollado iniciativas de reconocimiento empresarial y encuentros para emprendedores, como son: la novena edición del "Día de la Persona Emprendedora en Andalucía", la tercera edición de los "Premios Andalucía Emprende" con fases iniciales provinciales, cuatro encuentros interprovinciales de networking, la novena edición del Premio Emprendedor XXI y el primer Foro Internacional de Emprendimiento de Andalucía Emprende.

- Realización de programas específicos de fomento, creación y consolidación empresarial como son: 200 Planes Locales para Emprender; la primera y segunda edición, aunque ésta última sólo se ha iniciado en 2015, de las Lanzaderas de Empleo y Emprendimiento Solidario de Andalucía Emprende; Programa Internacional para Emprendedores; Proyecto Lunar; Andalucía Emprende Coopera; Aceleradora de Empresas (CelerAEmprende); el inicio de la red de centros de referencia para el fomento de la economía social; Programa Seniors; Proyecto Minerva; Iniciativa @mprende+; Programa 100 Caminos al Éxito; Andalucía Openfuture y la participación en diversos proyectos europeos. 
- El inicio de ocho proyectos de investigación sobre emprendimiento, dinamismo emprendedor y buenas prácticas.

- Contratación de una campaña de comunicación en medios de ámbito regional, difusión en medios de comunicación de experiencias emprendedoras, Andalucía Emprende TV, etc.

Por todo ello, el valor del análisis de nuestro estudio es menor, al que resultaría de haber podido incluir todas estas actividades con una valoración económica y haber incluido los efectos positivos de la actividad de Andalucía Emprende realizada durante el 2015 en años posteriores.

\section{4.- Discusión y conclusiones}

Existe una preocupación creciente, tanto en la sociedad, como en las Administraciones Públicas, por conocer la eficacia del empleo de los fondos públicos. Esta preocupación se ha acrecentado en los últimos años por la crisis existente y la consecuente necesidad de reducir las partidas de gasto públicas, lo que hace que se ejerza una mayor presión de contención del gasto y un mayor interés por la evaluación de los organismos que ejecutan las políticas públicas.

En la literatura, la falta de consenso ha provocado la existencia de una amplia gama de propuestas metodológicas para el estudio del impacto económico y social de las organizaciones. En el presente trabajo se ha desarrollado un análisis coste-beneficio para evaluar los retornos generados por Andalucía Emprende en la ejecución de las políticas de emprendimiento encomendadas por la Junta de Andalucía. Se ha iniciado el estudio con un análisis de los stakeholders, desde el punto de vista ontológico, en función del valor añadido, tanto monetario como no monetario, que reciben con la actividad de la fundación, obteniendo los outcomes generados en la actividad de la organización.

El resultado obtenido es que por cada euro invertido por la Administración en esta entidad, ésta devolvió a la Administración (tanto nacional como autonómica y local) y a la sociedad 6,75 euros en el año 2015, sin tener en cuenta la creación de valor de la actividad de 2015 en los años siguientes ni determinadas actividades realizadas en ese año que no han podido ser valoradas económicamente. A priori, resulta una buena gestión de los fondos públicos, ya que se genera una cifra muy superior a la invertida, sin entrar a valorar el coste de oportunidad que se pudiera dar si esos fondos se invierten en otro proyecto distinto.

La principal limitación de este estudio viene definida por las propias características del método empleado, por la dificultad de valorar algunos outcomes que genera la organización con su actividad, así como por la existencia de intangibles en los que no ha sido posible su monetización. 
Las futuras líneas de investigación deberían centrar su atención en la mejora metodológica que contribuya a consensuar un método que puedan utilizar las empresas de manera genérica y sistemática, como ocurre en contabilidad, con una serie de normas internacionales aceptadas por la comunidad científica que permita incluir en los balances de las empresas todo el valor social y medioambiental que generan.

\section{Bibliografía}

ADB (ASIAN DEVELOPMENT BANK) (2013): Cost-benefit analysis for development. A practical guide, Asian Development Bank. En: https://www.adb.org/sites/default/files/institutional-document/33788/iles/cost-benefit-analysis-development.pdf. Revisado en enero 2017.

ARVIDSON, A., LYON, F., MCKAY, S. \& MORO, D. (2010): "The ambitions and challenges of SROI", Third Sector Research Center, Working Paper 49.

AWASTHI, D.N. \& SEBASTIÁN, J. (1996): Evaluation of entrepreneurship development programmes, Sage Publications.

BAMBERGER, L. \& GROSS, C. (2007): "Count what counts: improving charitable investor access to the community development sector with better data and better analytical models", Investment Review, 64, 64-75.

BAUCHET, J. \& MORDUCH, J. (2013): "Is Micro too Small? Microcredit vs. SME Finance", World Development, 43, 288-297.

BREST, P., HARVEY, H. \& LOW, K. (2009): "Calculated Impact", Stanford Social Innovation Review, 7 (1), 50-56.

CHRISTOFFERSEN, S., FRAMPTON, G.C. \& GRANITZ, E. (2013): "Environmental sustainability's impact on earnings", Journal of Business \& Economics Research, 11 (7), 325-334.

CLARK, C., ROSENZWEIG, W., LONG, D. \& OLSEN, S. (2004): "Double bottom line project report: Assessing social impact in double bottom line ventures", Working Paper Series, $n^{\circ} 13$, University of California, Berkeley. En: http://www.riseproject.org/DBL_Methods_Catalog.pdf. Revisado en marzo 2016.

DAIGLE, J., HALL, C., JAMAL, R., SILVA-LEANDER, K. \& TAGAR, E. (2004): Poverty alleviation through socially responsible investment. Case studies of community investing and social venture capital, School of International and Public Affairs, Columbia University. 
DE PABLO VALENCIANO, J. \& URIBE TORIL, J. (2009): "Emprendimiento de la economía social y desarrollo local: la promoción de incubadoras de empresas de economía social en Andalucía", CIRIEC- España, Revista de Economía Pública, Social y Cooperativa, 64, 5-33.

DE PABLO VALENCIANO, J., URIBE TORIL, J. \& LEVY MANGIN, J.P. (2010): "The business school programme, within the framework of the territorial network of support to the entrepreneur in Andalusia (Spain)", iBusiness, 2, 326-332.

DYWAN, B., GILCHRIST, T. \& LOTFABADI, R. (2010): "The Stop: will work for food security", Business Modeling \& Policy Innovation, 3-21.

EIZAGUIRRE, S. (2016): "De la innovación social a la economía solidaria. Claves prácticas para el desarrollo de políticas públicas", CIRIEC-España, Revista de Economía Pública, Social y Cooperativa, 88, 201-230.

ESTEVES, A.M., FRANKS, D. \& VANCLAY, F. (2012): "Social impact assessment: the state of the art", Impact Assessment and Project Appraisal, 30 (1), 34-42.

FERNÁNDEZ BAPTISTA, A. (2012): "Los sistemas de gestión ética social y medioambiental como promotores del capital social, Telos: Revista de Estudios Interdisciplinarios en Ciencias Sociales, 13 (3), 312-328.

FLORMAN, M., KLINGLER-VIDRA, R. \& FACADA, M.J. (2016): "A critical evaluation of social impact assessment methodologies and a call to measure economic and social impact holistically through the External Rate of Return platform", Working Paper, 1602. LSE Enterprise, London.

GALLEGO, M. (2012): "El balance social como herramienta de auditoria organizacional", Revista Universidad EAFIT, 35 (115), 27-40.

GARRIGA, E (2016): Modelo de impacto social, IC Eada-Seres. En: http://www.fundacionseres.org/Lists/Herramientas/Attachments/14/IC\%20EADA\%20SERES\%202016.pd f. Revisado en enero 2017.

GRABENWARTER, U. \& LIECHSTENSTEIN, H. (2011): "In search of gamma: an unconventional perspective on impact investing", IESE working paper.

GRI (GLOBAL REPORTING INICIATIVE) (2016): Implementation manual, Global Reporting Iniciative. En: https://www.globalreporting.org/resourcelibrary/GRIG4-Part2-Implementation-Manual.pdf. Revisado en enero de 2017.

GRIECO, C. (2015): Assessing Social Impact of Social Enterprises, SpringerBriefs in Business.

GUZMÁN ALFONSO, C., SANTOS CUMPLIDO, F.J \& BARROSO GONZÁLEZ, M. (2016): "Cooperativismo, factor empresarial y desarrollo económico: propuesta de un modelo teórico de enlace", REVESCO, Revista de Estudios Cooperativos, 122, 110-134. 
HEHENBERGER, L., HARLING, A. \& SCHOLTEN, P. (2013): Practical guide to measuring and managing impact, European Venture Philanthropy Association.

HONEYMAN, R. (2014): The B Corp Handbook: How to Use Business as a Force for Good, BerrettKoehler Publishers.

JOHANNESSON, M. \& WEINSTEIN, M.C. (1993): "On the decision rules of cost-effectiveness analysis", Journal of Health Economics, 12, 459-467.

KAPLAN, R.S. \& NORTON, D.P. (1996): "Using the balanced scorecard as a strategic management system", Harvard Business Review, 74 (1), 75-85.

LAMBERTON, G. (2005): "Sustainability accounting-a brief history and conceptual framework", Accounting Forum, 29 (1), 7-26.

LITTLE, I. \& MIRRLEES, J. (1969): Manual of Industrial Project Analysis in Developing Countries, vol. 2, Organisation for Economic Co-operation and Development, Paris.

LÓPEZ, D., SÁNCHEZ, F., VIDAL, E., PEGUEROLES, J., ALIER, M., CABRÉ, J., GARCÍA, J. \& GARCÍA, H. (2014): "A methodology to introduce sustainability into the final year project to foster sustainable engineering projects". En: Frontiers in Education Conference (FIE), IEEE.

LÓPEZ ÁLVAREZ, N. \& BLANCO ERAS, D. (2008): "Metodología para el Cálculo de la huella ecológica en universidades". En: Congreso Nacional del Medio Ambiente, Cumbre del Desarrollo Sostenible, Madrid.

LUMLEY, T., LANGERMAN, C. \& BROOKES, M. (2005): Funding success, New Philanthropy Capital. En: http://www.thinknpc.org/wp-content/uploads/2012/09/Funding-success-print-version.pdf. Revisado en enero 2017.

MAAS, K. (2014): "Classifying social impact measurement frameworks", The Conference Board Giving Thoughts series, 1 (2). En: https://www.conference-board.org/retrievefile.cfm?filename=TCB_GTV1N2-141.pdf\&type=subsite. Revisado en marzo 2016.

MAAS, K. \& LIKET, K. (2011): "Social impact measurement: classification of methods". En Burritt, R.L., Schaltegger, S., Bennett, M., Pohjola, T., Csutora, M. (Eds.): Environmental Management Accounting and Supply Chain Management, Springer.

MARTÍ-COSTA, M. (Dir.) (2010): Políticas públicas de promoción de cooperativas en España. Una visión comparada entre comunidades autónomas, Institut de Govern i Polítiques Públiques.

MOFFATT, I. (2000): "Ecological footprints and sustainable development", Ecological Economics, 32, 359-362.

MOZAS MORAL, D. \& BERNAL JURADO, E. (2007): "La economía social como generadora de cohesión y empleo: el caso andaluz". En: Salinas Ramos, F. \& Herranz De La Casa, J.M., La economía social, instrumento de cohesión y empleo en Castilla y León, Universidad Católica de Ávila. 
MULGAN, G. (2010): "Measuring social value", Standfor Social Innovation Review, 8 (3), 38-43.

OLSEN, S. \& GALIMIDI, B. (2008): Catalog of approaches to impact measurement, Social Venture Technology Group.

OLSEN, S. \& NICHOLLS, J. (2005): "A Framework for Approaches to SROI Analysis." En: https://ccednet-rcdec.ca/sites/ccednet-rcdec.ca/files/ccednet/pdfs/2005-050624_SROI_Framework.pdf. Revisado en enero 2017.

OSPINA BOZO, S. (2001): "Evaluación de la Gestión Pública: Conceptos y aplicaciones en el Caso Latinoamericano", Revista CLAD Reforma y Democracia, 19, Caracas.

PÉREZ GONZÁLEZ, M.C. \& VALIENTE PALMA, L. (2017): "La localización sectorial del cooperativismo: una aproximación a nivel territorial español", REVESCO, Revista de Estudios Cooperativos, $123,198-224$.

PINTO, J.L., SACRISTÁN, J.A. \& ANTOÑANZAS (2008): "Reflexiones sobre reglas de decisión, costeefectividad e impacto presupuestario", Gaceta Sanitaria, 22 (6), 585-589.

PLANAS MIRET, I. (2005): "Principales mecanismos de evaluación económica de políticas públicas", Ekonomiaz, 1 (60), 98-121.

REEDER, N. \& COLANTONIO, A. (2013): "Measuring impact and non-financial returns in impact investing: a critical overview of concepts and practice", EIBURS Working Paper 2013/01, London School of Economics and Political Science. En: https://files.Isecities.net/files/2013/10/Measuring_Impactfull-length-Oct-20131.pdf. Revisado septiembre 2016.

RETOLAZA AVALOS, J.L., SAN JOSE RUIZ DE AGUIRRE, L. \& RUIZ-ROQUEÑI, M. (2016): Social accounting for sustainability: monetizing social value, Springer.

RETOLAZA AVALOS, J.L., SAN JOSE RUIZ DE AGUIRRE, L. \& RUIZ-ROQUEÑI, M. (2014): "Ontological stakeholder view: an innovative proposition", Global Business Review, 15 (1), 25-36.

SCHOENJAHN, A. (2012): "New faces of corporate responsibility: Will new entity forms allow businesses to do good?", Journal of Corporation Law, 37 (2), 453-473.

STUFFLEBEAM, D.L. \& SHINKFIELD, A.J. (1993): Evaluación sistemática. Guía teórica y práctica, Ediciones Paidós, Barcelona.

TRELSTAD, B. (2008): "Simple measures for social enterprise", Innovations, 3 (3), 105-118.

TRUCOST (2015): Trucost's valuation methodology. En: http://www.gabisoftware.com/fileadmin/GaBi_Databases/Thinkstep_Trucost_NCA_factors_methodology_report.pdf. Revisado en enero 2017.

TUAN, M.T. (2008): "Measuring and/or estimating social value creation: insights into eight integrated cost approaches", Bill y Melinda Gates Foundation, Final Paper. En: https://docs.gatesfoundation.org/documents/wwl-report-measuring-estimating-social-value-creation.pdf. Revisado en febrero 2017. 
VALLECILLO, M.R. \& GUTIÉRREZ, J.J. (2016): "La responsabilidad social pública como elemento de política de empleo", CIRIEC-España, Revista de Economía Pública, Social y Cooperativa, 87, 1138.

VEDUNG, E. (1997): Evaluación de políticas públicas y programas, Ministerio de Trabajo y Asuntos Sociales, Madrid.

VELDMAN, P. (2009): "Learning social metrics from international development", World Bank Research Observer, 24 (1), 71-113.

WIESNER DURÁN, E. (2000): Función de evaluación de planes, programas, estrategias y proyectos, Gestión Pública Serie $n^{\circ} 4$, Instituto Latinoamericano y del Caribe de Planificación Económica y Social, Santiago de Chile.

WOOD, C. \& LEIGHTON, D. (2010): Measuring social value the gap between policy and practice, Demos. En: https://www.demos.co.uk/files/Measuring_social_value_-_web.pdf?1278410043. Revisado en enero de 2017.

\section{Referencias páginas web}

AGENCIA TRIBUTARIA (2016): Informe anual de recaudación tributaria. Año 2015. En: http://www.agenciatributaria.es/static_files/AEAT/Estudios/Estadisticas/Informes_Estadisticos/Informes_A nuales_de_Recaudacion_Tributaria/Ejercicio_2015/IART15.pdf. Revisado en enero 2017.

ANDALUCÍA EMPRENDE, FUNDACIÓN PÚBLICA ANDALUZA (2015a): Informe de supervivencia empresarial 2008-2014. En: http://www.andaluciaemprende.es/wpcontent/uploads/2015/02/Informe_Supervivencia_2008-2014_Vd.pdf

ANDALUCÍA EMPRENDE, FUNDACIÓN PÚBLICA ANDALUZA (2015b): Memorias de actividades 2010-2014. En: https://www.andaluciaemprende.es/publicaciones-y-estadisticas/resultados-deactividad-y-memorias/.

ANDALUCÍA EMPRENDE, FUNDACIÓN PÚBLICA ANDALUZA (2016): Informe de gestión 2015. En: http://www.andaluciaemprende.es/wp-content/uploads/2015/02//NFORME-DE-GESTI\%C3\%93NANDALUC\%C3\%8DA-EMPRENDE-2015-web.pdf. Revisado en noviembre de 2016.

CÁMARA DE COMERCIO DE TOLEDO (2017): Viveros de empresa. En: http://www.viveroscamaratoledo.com/tarifas/. Revisado en enero 2017.

CENTER FOR HIGH IMPACT PHILANTHROPY (2017): Linking Cost and Impact. En: http://www.impact.upenn.edu/our-analysis/how-can-i-practice-high-impactphilanthropy/linking_cost_and_impact/. Revisado en enero 2017. 
COLEGIO DE ECONOMISTAS DE VALLADOLID (2005): Honorarios profesionales orientativos. En: http://www.ecova.es/docs/8_tarifas_00_euros.pdf. Revisado en enero 2017.

CONSEJERÍA DE HACIENDA Y ADMINISTRACIÓN PÚBLICA (2016): Cuentas anuales 2015 Fundación Pública Andaluza Andalucia Emprende. En: https://www.juntadeandalucia.es/haciendayadministracionpublica/servicios/fiscalizacion/rendicion/cuenta2015/cuenta/fundaciones.htm. Revisado en diciembre 2016.

DALBERG (2017): Global Development Advisors. En: http://www.dalberg.com/. Revisado en enero 2017.

FORÉTICA (2017): SGE-21 Sistema de Gestión Ética y Socialmente Responsable. En: http://www.foretica.org/wp-content/uploads/2016/01/SGE-21_2008_ES-DEF.pdf. Revisado en enero 2017.

FOUNDATION CENTER (2017): Movement Above the U.S. \$1 a Day Threshold. En: http://trasi.foundationcenter.org/record.php?SN=89. Revisado en enero 2017.

HIPINVESTOR (2017): Human impact + profit investor. En: http:/hipinvestor.com/ . Revisado en febrero 2017.

IAAP, INSTITUTO ANDALUZ DE ADMINISTRACIONES PÚBLICAS (2013): Circular, 01/2013 de 18 de enero, de la dirección del instituto andaluz de administración pública, para la gestión de acciones formativas. En:

http://www.juntadeandalucia.es/institutodeadministracionpublica/publico/anexos/masiaap/Circula r_18Enero2013.pdf. Revisado en enero 2017.

IEA (2016a): Actividad financiera y empresarial. Actividad empresarial. Cuenta de pérdidas y ganancias de las empresas de Andalucía por provincia. Año 2014. En: $\mathrm{https}: /$ www.google.es/url?sa=t\&rct=j\&q=\&esrc=s\&source=web\&cd=1\&ved=0ahUKEwj3_lyssdPTAhWBv BQKHcOvAooQFggkMAA\&url=http\%3A\%2F\%2Fwww.juntadeandalucia.es\%2Finstitutodeestadisticayca rtografia\%2Fiea\%2Fpublicaciones\%2Fdatos\%2FAnuarioEstadisticoAndalucia\%2FCapitulo12\%2Fanuari 0120208.xls\&usg=AFQjCNH08foW4O2qRoOhfSJWAmaljfgKLw\&cad=rja. Revisado en enero 2017.

IEA (2016b): Anuario Estadístico de Andalucía. Ingresos y gastos de los hogares. Prestaciones sociales económicas. Pensión media anual según sexo por provincia. En: $\mathrm{http} / /$ www.juntadeandalucia.es/institutodeestadisticaycartografia/iea/resultadosConsulta.jsp?CodOper=6 4\&codConsulta=92729. Revisado en enero 2017.

IEA (2016c): Anuario Estadístico de Andalucía. Ingresos y gastos de los hogares. Ingresos del trabajo. Asalariados, percepciones salariales y salarios según sexo por provincia. En: http://www.juntadeandalucia.es/institutodeestadisticaycartografia/iea/resultadosConsulta.jsp?CodOper=64\&codConsult a=92717. Revisado en enero 2017. 
IEA (2016d): Estadísticas de Actividad empresarial. Demografía de empresas. Demografía de empresas y empleo por estrato de empleo. Año 2015. En: https:/www.google.es/url?sa=t\&rct=j\&q=\&esrc=s\&source=web\&cd=4\&ved=0ahUKEwiqidaZstPTAhXLv xQKHR7yBpkQFgg2MAM\&url=http\%3A\%2F\%2Fwww.juntadeandalucia.es\%2Finstitutodeestadisticayc artografia\%2Fdemogemp\%2Ftab\%2F2015\%2Ftab\%2FDEMP_15_24.xls\&usg=AFQjCNEB8ty3D64ZU pr2g1-xHGW718Nu2w\&cad=rja. Revisado en enero 2017.

IEA (2016e): Indicadores Andalucía y Provincias. Mercado de trabajo. En: http://www.juntadeandalucia.es/institutodeestadisticaycartografia/28Febrero/tema.htm?t=merctrab\&i=03070\&m=s\#indice. Revisado en enero 2017.

JUNTA DE ANDALUCÍA (2017): Anteproyecto de Ley Andaluza de Fomento del Emprendimiento. En: http://www.juntadeandalucia.es/expor/drupalida/Anteproyecto\%20de\%20Ley\%20de\%20Fomento\%20d el\%20Emprendimiento.pdf. Revisado en enero 2017.

LBG (2017a): The global standard for measuring corporate community investment. En: http://www.lbgonline.net/\#. Revisado en enero 2017.

LBG (2017b): La metodología para la medición, gestión y comunicación de la acción social empresarial. En: https://www.lbg.es/es/home. Revisado en enero 2017.

MINISTERIO DE EMPLEO Y SEGURIDAD SOCIAL (2015): Orden ESS/86/2015, de 30 de enero, por la que se desarrollan las normas legales de cotización a la Seguridad Social, desempleo, protección por cese de actividad, Fondo de Garantía Salarial y formación profesional, contenidas en la Ley 36/2014, de 26 de diciembre, de Presupuestos Generales del Estado para el año 2015. En: https://www.boe.es/boe/dias/2015/01/31/pdfs/BOE-A-2015-847.pdf. Revisado diciembre 2016.

PROGRESS OUT OF POVERTY (2017): About the PPI: a poverty measurement tool. En: http://www.progressoutofpoverty.org/about-ppi. Revisado en enero 2017. 